\title{
Glosoplastía con Técnica de Harada en un Paciente con Síndrome de Down
}

\author{
Glossoplasty with Harada's Technique in a Patient with Down Syndrome
}

Araceli Raposo*; Günther Preisler, ${ }^{*, *}$; Fernando Salinas ${ }^{* *}$ \& Carlos Muñoz

\begin{abstract}
RAPOSO, A.; PREISLER, G.; SALINAS, F. \& MUÑOZ, C. Glosoplastía con técnica de Harada en un paciente con Síndrome de Down. Int. J. Odontostomat., 5(3):245-248, 2011.

RESUMEN: La macroglosia es una patología que afecta frecuentemente a los niños que padecen síndrome de Down, lo cual condiciona que la lengua siempre se encuentre expuesta al medio externo y sufra marcada sequedad. Por estas razones se ha señalado que al tratar quirúrgicamente la macroglosia existente no solo mejoraría del aspecto estético y la aceptación social, sino que funcionalmente mejora el lenguaje, el desarrollo mandibular, la mordida abierta, y ocurre espontáneamente la recolocación del arco dental. Así se han creado y modificado una serie de técnicas quirúrgicas para reducir el tamaño de la lengua. En el artículo se presenta un caso clínico de una paciente con síndrome de Down que fue sometida a una glosoplastía con la técnica de Harada. Se discute los beneficios de las glosoplastías, así como las diferentes técnicas empleadas para ello.
\end{abstract}

PALABRAS CLAVE: macroglosia, tratamiento, reducción.

\section{INTRODUCCIÓN}

La macroglosia se define tradicionalmente como una lengua en reposo que sobresale más allá de los dientes o reborde alveolar. El diagnóstico se basa generalmente en este signo y la comparación con una lengua aparentemente normal (mediciones objetivas del tamaño no son confiables) (Murthy \& Laing, 1994).

Esta afección puede ser clasificada como macroglosia verdadera, que se asocia a observaciones histopatológicas definitivas, o pseudomacroglossia, que es una ampliación de la relación de la lengua secundaria a una pequeña mandíbula y sin alteraciones histológicas demostrables. La macroglosia verdadera puede ser primaria, caracterizada por hipertrofia o hiperplasia de los músculos de la lengua, o secundaria, resultado de la infiltración de tejido normal con elementos anómalos (Murthy \& Laing).

La pseudomacroglosia es una condición en que la lengua es normal en tamaño pero es relativamente grande con respecto a sus relaciones anatómicas; puede ser debido a postura habitual de la lengua, hi- pertrofia tonsilar y de adenoides, quistes o tumores que desplazan la lengua hacia adelante, paladar bajo, y diferencia de los arcos superior e inferior en el plano transversal, vertical y anteroposterior, así como el micrognatismo inferior (Martinez, 2006).

En términos de frecuencia las causa habituales de macroglosia verdadera son; hipertrofia muscular, síndrome de Down, linfangioma, angiomas y fibromas (Martinez).

La pseudomacroglosia suele ser autolimitada y regresiona con la edad. La ampliación aparente de la lengua secundaria a micrognatia se observa en el síndrome de Down y en el síndrome de Pierre-Robin y en ocasiones en condiciones con disfunción (Murthy \& Laing).

La cavidad bucal de los niños con trisomía 21 es pequeña y tiende a mantenerse siempre abierta debido a la hipotonía muscular. El paladar duro es corto y la mandíbula muestra tendencia a clase III de Angle,

\footnotetext{
* Departamento de Cirugía Buco-maxilofacial, Universidad Austral de Chile, Valdivia, Chile.

* Servicio de Cirugía Buco-maxilofacial Hospital Base de Valdivia, Valdivia, Chile.
} 
presentan varias desarmonías oclusales y la lengua es macroglósica, de aspecto escrotal, que protruye a través de labios carnosos, por lo que el piso bucal y el diámetro anteroposterior de la bucofaringe están disminuidos con respecto al tamaño lingual, lo cual condiciona que ésta siempre se encuentre expuesta al medio externo y sufra marcada y permanente sequedad, agravada por la succión lingual, la boca siempre abierta y la respiración de tipo bucal. Por estas razones se ha señalado en algunos reportes que al tratar quirúrgicamente la macroglosia existente no solo mejoraría del aspecto estético y la aceptación social, sino que funcionalmente mejora el lenguaje, el desarrollo mandibular, la mordida abierta, y ocurre espontáneamente la recolocación del arco dental. Mejora la masticación, deglución y se logra un incremento en el tamaño del pasaje de la vía aérea y por tanto una disminución de las infecciones bucales y respiratoria (López, 2007).

El propósito de este artículo es describir un caso clínico, revisar brevemente las distintas técnicas de glosoplastías y discutir los beneficios de éstas.

\section{REPORTE DEL CASO}

Paciente sexo femenino, con síndrome de Down, antecedentes de cardiopatía congénita tratada y asma controlado, es derivada en agosto del 2010 al servicio de patología bucal y maxilofacial del Hospital Base de Valdivia, por presentar pseudomacroglosia con lesión ulcerada de 3 meses de evolución en el tercio posterior del borde izquierdo de la lengua y en cara ventral, con impronta de dientes superiores. La lesión presenta bordes indurados y queratinizados. La paciente relata dolor de mediana intensidad y dificultad para la ingesta de alimentos. Se toma biopsia incisional, que arroja como resultado úlcera inflamatoria inespecífica compatible con úlcera traumática.

Paciente es evaluada en el servicio de cirugía oral y maxilofacial del mismo hospital donde se decide realizar la glosectomía parcial utilizando la técnica de Harada. En Septiembre del mismo año se realiza la cirugía bajo anestesia general. Primero se procede a marcar las incisiones que se realizarán, en forma de una cuña en el centro de la lengua y una forma de media luna en la parte posterior del dorso (Fig. 1). La combinación de estos dos tipos de incisiones, resulta en la reducción en sentido longitudinal y transversal. La herida quirúrgica se sutura por planos (Fig. 2). Fi- nalmente se realizan controles periódicos hasta los 6 meses postoperatorios aproximadamente (Fig. 3).

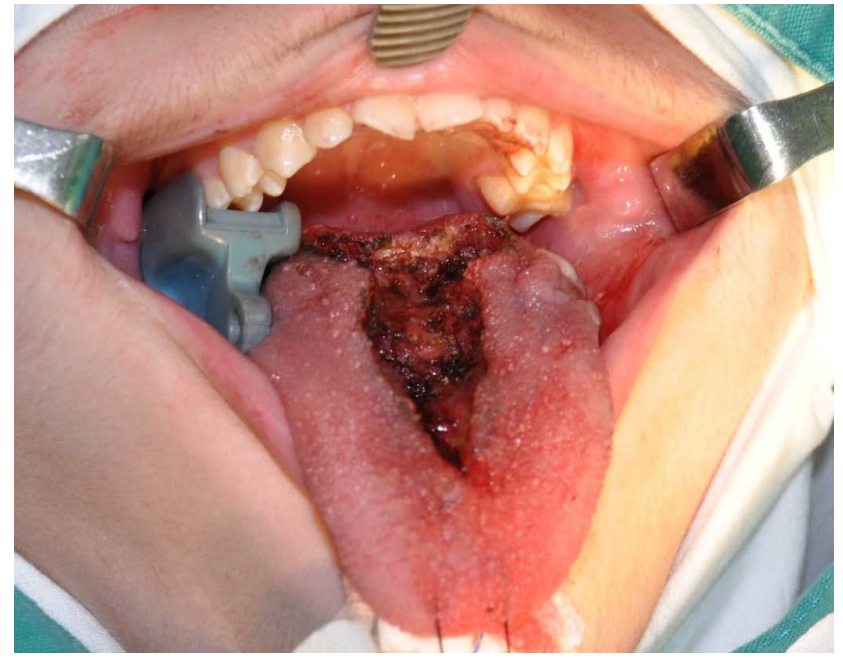

Fig. 1. Incisiones con técnica de Harada realizadas con electrobisturí.

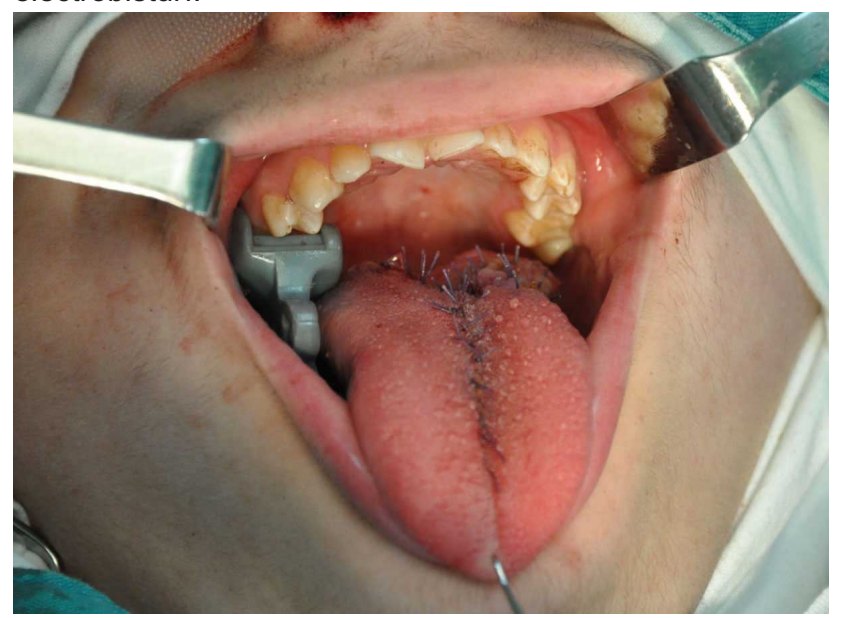

Fig. 2. Sutura por planos, muestra la reducción tanto en ancho como en largo de la lengua.

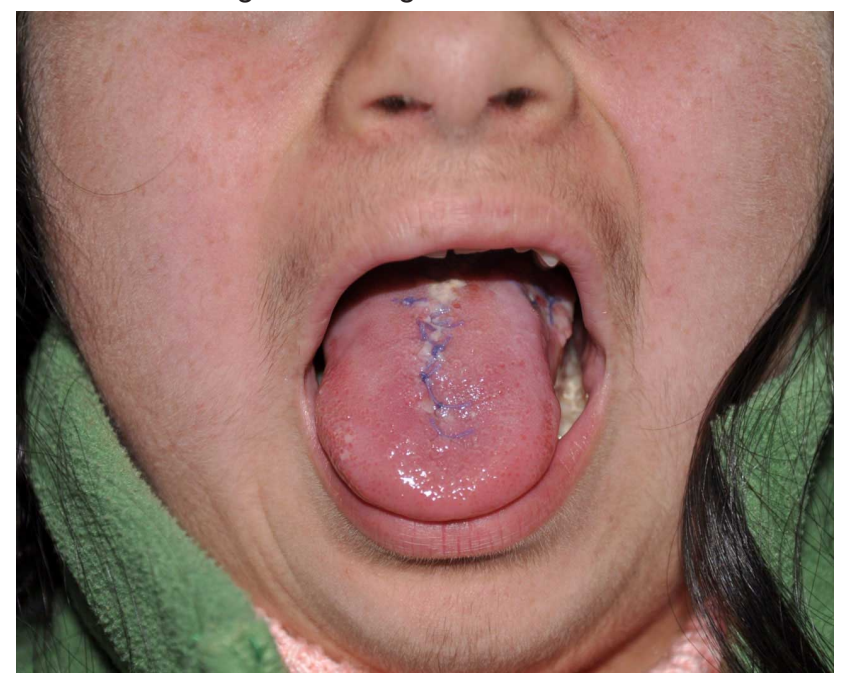

Figura 3. Control postoperatorio a los 7 días. 


\section{DISCUSIÓN}

Varias técnicas se describen para reducir el volumen de la lengua, que van desde la eliminación de la parte anterior o central de la lengua a la extirpación completa de los márgenes anterior y lateral de la lengua. Sin embargo el tratamiento continúa siendo controversial, por la falta de acuerdo en los criterios diagnósticos y la falta de estudios longitudinales que comparen los resultados a largo plazo de las técnicas para corregir esta condición (latrou et al., 2008). Para determinar si la glosectomía parcial es un procedimiento necesario, es importante identificar los signos y síntomas de la macroglosia; no todas las características están siempre presentes y su existencia no es de necesidad un signo patognomónico para el diagnóstico de la macroglosia (Martinez). Los criterios clínicos que se consideran como indicadores de glosoplastía son la morfología (discrepancia de tamaño entre la lengua y la cavidad oral), protrusión lingual, problemas dentoesqueletales (prognatismo mandibular, mordida abierta anterior, inclinación excesiva de los incisivos inferiores) y deficiencias funcionales (dificultad para tragar, respirar o articular, y constante babeo (latrou et al.; Wolford \& Cottrell, 1996). Además se ha considerado las consecuencias psicológicas por la apariencia del paciente como un factor a tener en cuenta al indicar la cirugía (Martinez).

La glosoplastía de reducción puede mejorar de forma significativa los resultados morfológicos y funcionales. Una mejora relativa de la función aérea el habla y la masticación puede ser anticipada. Si la macroglosia es verdaderamente causante de un crecimiento mandibular desfavorable, la reducción de la lengua puede contribuir o controlarlo (Díaz et al., 2006).

Un estudio realizado en Cuba el año 2007, comparó la incidencia de infecciones bucales y respiratorias, antes y después de la glosectomía parcial en niños con síndrome de Down, observando una disminución significativa de éstas. Se demostró el valor del tratamiento quirúrgico de la macroglosia en la mejora de la calidad de vida del niño con esta patología (López).

Respecto a las técnicas de glosectomías, se han descrito varias formas de realizar el procedimiento (Wolford \& Cottrell). En un principio las técnicas reducían en largo pero no en ancho, pero las distintas modificaciones han solucionado este problema. Por otra parte estas variaciones de las técnicas apuntan a minimizar las consecuencias de la cirugía pero en gene- ral todas las técnicas tenían la misma desventaja, después de la operación donde el paciente pierde la punta de la lengua, se altera la parte más sensitiva somato sensorial y gusto (Kruchinsky, 2006).

Kruchinsky presenta un nuevo método con mejores resultados funcionales al preservar la sensación somática y gusto al mantener los receptores del gusto de la punta de la lengua, sin embargo esta técnica no es sencilla ya que las incisiones que implica no son fáciles de realizar.

Autores como Wolford \& Cottrell señalan que la resección anterior combinado con la línea media procedimiento de tipo ojo de cerradura es la mejor técnica, técnica que según Kruchinsky tendría la desventaja de perder parte de la capacidad somatosensorial de la lengua, sin embargo estos autores señalan que ellos no reportaron alteraciones del gusto ni de la movilidad (Wolford \& Cottrell).

La técnica de Harada, descrita en 1995, consiste en realizar una línea en forma de cuña en el centro de la lengua, y una forma de media luna en la parte posterior del dorso. La combinación de estos dos, resulta en la reducción en sentido longitudinal y transversal. Otra ventaja es que con esta técnica se puede minimizar la forma de oreja de perro. Por otro lado se resuelve los problemas relacionados a la perdida de la punta de la lengua. Las incisiones son muy simples y las funciones de la lengua (habla, movilidad y gusto) por lo general permanecen normales después de la cirugía (Harada \& Enomoto, 1995).

Lamentablemente no existen estudios que comparen entre dos o más técnicas, pero como cualquier medida terapéutica, una solución no puede ser aplicada a todas las situaciones, por lo tanto, estas deben ser adaptadas a las necesidades específicas del paciente (Rizer et al., 1985).

De esta manera se concluye que son muchos los beneficios de las glosoplastías en pacientes con necesidades especiales como los pacientes con síndrome de Down, obteniéndose buenos resultados desde el punto de vista estético, funcional y social. La técnica de Harada es una técnica relativamente fácil de realizar y que a diferencia de otras reduce en longitud y en ancho el tamaño de la lengua, sin provocar daños en la funcionalidad de ésta. 
RAPOSO, A.; PREISLER, G.; SALINAS, F. \& MUÑOZ, C. Glossoplasty with Harada's technique in a patient with Down Syndrome. Int. J. Odontostomat., 5(3):245-248, 2011.

ABSTRACT: Macroglossia is a condition that often affects children with Down syndrome, it makes the tongue to always be exposed to the environment and suffer noticeable dryness. For these reasons it has been demonstrated that macroglossia treated surgically not only improves the esthetic aspect and the social acceptance, but functionally improves language, development of the mandible, and open bite, with repositioning of the dental arch occurring spontaneously. Many surgical techniques have been created and modified to reduce the size of the tongue. In this article we present the case of a patient with Down syndrome who underwent a glossoplasty with the Harada's technique. The benefits of the glossoplasties, as well as the different techniques used for it are discussed.

KEY WORDS: macroglossia, treatment, reduction.

\section{REFERENCIAS BIBLIOGRÁFICAS}

Díaz, J.; Jardón, J.; Quintero, Y.; Peña, M. \& Roca, C. Macroglosia asociada con deformidades dentofaciales: Alternativas terapéuticas y presentación de un caso. Rev. Cubana Estomatol., 43(4), 2006. Disponible en: http:// scielo.s/d.cu/scielo.php?script=sci_arttext\&pid=S0034$75072006000400007 \&$ Ing=es.

Harada, K. \& Enomoto, S. A new method of tongue reduction for macroglossia. J. Oral Maxillofac. Surg., 53:91-2, 1995.

latrou, I. A.; Schoinohoriti, O. K.; Tzerbos, F. \& Pasparakis, D. Treatment of macroglossia in a child with Weaver síndrome. Int. J. Oral Maxillofac. Surg., 37:961-5, 2008.

Kruchinsky, H. V. Once Again About a New Tongue Reduction Method. J. Oral Maxillofac. Surg., 64:1696-700, 2006.

López, Z. Glosectomía parcial en la trisomía 21: incidencia de las infecciones bucales y respiratorias, preoperatorias y posoperatorias. Rev. Cubana Pediatr., 79, 2007. Disponible en: http://scielo.s/d.cul scielo.php?script=sci_arttext\&pid=S0034$75312007000300007 \&$ lng $=\bar{e}$.

Martinez, L. P. Macroglossia: etiología multifactorial, manejo múltiple. Colomb. Méd., 37:67-73, 2006.

Murthy, P. \& Laing, M. R. Macroglossia. BMJ, 309:1386-7, 1994.

Rizer, F. M.; Schechter, G. L. \& Richardson, M. A. Macroglossia: etiologic considerations and management techniques. Int. J. Pediatr. Otorhinolaryngol., 8:225-36, 1985.

Wolford, L. M. \& Cottrell, D. A. Diagnosis of macroglossia and indications for reduction glossectomy. Am. J. Orthod. Dentofacial Orthop., 110:170-7, 1996.
Dirección para correspondencia:

Araceli Raposo

Departamento de Cirugía Buco-maxilofacial

Universidad Austral de Chile

Valdivia

CHILE

Email: araceliraposo@gmail.com

Recibido : 13-10-2011

Aceptado: 12-11-2011 